\title{
BMJ Open Efficacy of advice from healthcare professionals to pregnant women on avoiding constrictive clothing around the trunk: a study protocol for a randomised controlled trial
}

\author{
Kenji Takehara, ${ }^{1}$ Sachiko Kato, ${ }^{2}$ Aiko Sasaki, ${ }^{3}$ Seung Chik Jwa, ${ }^{3}$ Naoko Kakee, ${ }^{4}$ \\ Haruhiko Sago, ${ }^{3}$ Yuko Noguchi, ${ }^{2}$ Tomoko Aoki, ${ }^{2}$ Eisuke Inoue,${ }^{5}$ Chieko Nitta, ${ }^{2}$ \\ Yumiko Ishii ${ }^{2}$
}

To cite: Takehara K, Kato S, Sasaki A, et al. Efficacy of advice from healthcare professionals to pregnant women on avoiding constrictive clothing around the trunk: a study protocol for a randomised controlled trial. BMJ Open 2015;5: e008252. doi:10.1136/ bmjopen-2015-008252

- Prepublication history for this paper is available online. To view these files please visit the journal online (http://dx.doi.org/10.1136/ bmjopen-2015-008252).

Received 24 March 2015 Revised 4 September 2015 Accepted 14 September 2015

CrossMark

For numbered affiliations see end of article.

Correspondence to Dr Kenji Takehara; takehara-k@ncchd.go.jp

\section{ABSTRACT}

Introduction: As a component of midwife care, eliminating clothing that constricts the trunk has been shown to markedly elevate the uterine fundus, soften the uterus and abdomen, and reduce the abdominal wall tension in women admitted to hospital due to the risk of miscarriage or premature delivery. However, no prospective study has conclusively verified the efficacy of avoiding constrictive clothes around the trunk in pregnant women. We aim to verify the efficacy of instructing pregnant women to wear loose clothing that does not constrict the trunk to reduce the risk of premature birth and improve quality of life (QoL) during pregnancy.

Methods and analysis: We will conduct a randomised controlled trial of pregnant women scheduled to deliver at the National Center for Child Health and Development in Tokyo, Japan. A total of 616 pregnant women, from whom written informed consent will be obtained, will be allocated randomly to an intervention group or a control group. Women in the control group will be provided with anaemia prevention leaflets at 20 weeks' gestation and skin-care leaflets at 30 weeks' gestation. Women in the intervention group will be provided with the same leaflets and will also receive health advice from health professionals to avoid constrictive clothing around the trunk. The primary outcome will be a difference between these groups in the frequency of any one of the following category variables: (1) cervical length $<30 \mathrm{~mm}$ up to 28 weeks' gestation, (2) hospital admission for threatened premature delivery, or (3) premature delivery. Secondary outcomes will include QoL during pregnancy, maternal state of health, and status of fetal development.

Ethics and dissemination: The Institutional Review Board and Ethics Committee at the National Center for Child Health and Development, Japan, has approved this study. Our findings will be widely disseminated through conference presentations and peer-reviewed publications.

Trial registration number: UMIN000016853.
Strengths and limitations of this study

- In this study, healthcare professionals will develop an advice programme for pregnant women on suitable types of clothing to wear and how to wear clothing comfortably during pregnancy. The study will verify the programme's efficacy in reducing the risk of premature birth and improving the quality of life during pregnancy.

- Compared with current premature delivery prevention programmes, this programme will be non-invasive, economical and easily provided without the need for special equipment.

- There is limited information on similar interventions in previous studies, and the evidence used for parameters such as sample size calculations may be inadequate.

- Members of the study staff who will implement the intervention, as well as participants, cannot be blinded.

\section{INTRODUCTION}

Preventing premature delivery and improving women's quality of life (QoL) both before and after childbirth are important issues in perinatal medicine. With the incidence of premature delivery at $7.5 \%$ in developed countries and $9.6 \%$ worldwide, ${ }^{1}$ the cultivation of interventional methods to prevent premature delivery is much needed. Randomised controlled trials (RCTs) and meta-analyses of these methods have already been performed whether preventive interventions such as therapy with magnesium and other supplements, ${ }^{2}$ drug therapy with medications such as terbutaline (a sympathetic $\beta 2$-receptor agonist), ${ }^{3}$ cervical cerclage, ${ }^{4}$ bed rest $^{5}$ and relaxation are effective. ${ }^{6}$ Thus far, no study has demonstrated the 
effectiveness of advice from healthcare professionals to pregnant women regarding the avoidance of wearing tight clothing that constricts the trunk area during pregnancy.

A woman's body undergoes many changes due to hormonal effects during pregnancy to support the uterus. Hormonal activity loosens up ligaments, and increases the capacity of the chest and abdominal cavity. The wearing of tight clothes can therefore interfere with these physical changes and cause discomfort. According to some midwives and obstetricians at our hospital, many pregnant Japanese women tend to choose tighter, more fashionable clothes over looser, more comfortable options. Women may be unaware that tight clothing can constrict their growing body. Even maternity wear, such as maternity pants, belly bands, brassieres and abdominal support, if tightly fitted, may constrict a pregnant woman's changing shape. Previous studies on the effect of clothing on health in ordinary adults have found that clothing-induced constriction may increase the time taken for faeces to pass through the intestines and decrease the amount of faeces, ${ }^{7}$ worsen autonomic nervous system function, ${ }^{8}$ worsen food digestive function, ${ }^{9}$ decrease trunk musculature activity and increase the risk of lumbar problems. ${ }^{10}$ Constipation is one of the most common minor problems experienced by pregnant women, ${ }^{11}$ and tight clothing may further aggravate this condition. ${ }^{7}$

It has also been suggested that hiesho ('sensitivity to the cold' $)^{12}$ - a concept widely accepted in Japan and elsewhere in Asia-is a risk factor for premature delivery. Previous studies have shown that pregnant women with hiesho have a 3.4-3.5 times higher risk for premature delivery compared to pregnant women without hiesho. ${ }^{13}{ }^{14}$ Advice from healthcare professionals on wearing clothes to prevent feeling cold has been found to be effective. ${ }^{15}$ Constriction from tight clothes may lead to hiesho due to restricted blood circulation. Thus, a physiological association of the pressure from clothing around the trunk with premature delivery and reduced QoL during pregnancy is certainly feasible.

The above studies suggest that a focus on clothing during pregnancy may be extremely important. However, previous studies on the types of clothing suitable for pregnant women and ways of wearing clothes comfortably during pregnancy have been limited, and have mostly focused on aspects such as the effectiveness of thigh-length support stockings to improve haemodynamic response. ${ }^{16}$ In addition, previous research has shown that the effectiveness of abdominal decompression on normal pregnant women is unclear. ${ }^{17} 18$ Overall, available evidence on the effect of clothing and abdominal compression during pregnancy is currently inadequate.

Between April 2011 and March 2013, we conducted a trial programme for pregnant women admitted to the National Center for Child Health and Development for threatened miscarriage or premature birth, where they were advised by healthcare professionals to reduce the pressure of their clothing around the trunk area. We then performed a retrospective study using data from the medical records of 208 women. ${ }^{19}$ We found that after adjustment for age, pre-pregnancy body mass index, delivery history, history of threatened miscarriage or premature birth, year of hospitalisation and prescription medications, the incidence of premature birth at $<34$ weeks' gestation was significantly lower in the intervention group (adjusted OR 0.16, 95\% CI 0.04 to 0.60 ). There was no association, however, with premature birth at $<37$ weeks' gestation, for which the adjusted OR was 0.72 (95\% CI 0.30 to 1.72$).{ }^{19}$ The results of this preliminary study provided important evidence to support the implementation of a clinical trial of an advice programme by healthcare professionals; however, the preliminary study had some limitations.

In the present study, we aim to verify the effectiveness of advice from healthcare professionals to pregnant women to avoid wearing trunk-constricting clothing in order to reduce the risk of premature birth and improve QoL during pregnancy.

\section{METHODS}

\section{Study design}

This study will be a single-blinded RCT. Figure 1 shows the study design in detail. It will be implemented at the National Center for Child Health and Development in Japan.

\section{Participants and recruitment}

The participants of this study will be pregnant women scheduled to give birth at the facility where the study will be implemented, and to whom none of the following 11 exclusion criteria apply: (1) individuals who undergo their first prenatal check-up after 20 weeks' gestation or later; (2) individuals with psychiatric conditions; (3) individuals with uterine deformity; (4) individuals with previous uterine surgery (conisation/ radical trachelectomy for cervical cancer); (5) individuals who have undergone circumferential suture of the cervix; (6) individuals with fetal diseases; (7) individuals with suspected recurrent pregnancy loss; (8) individuals whose cervical length is already $<30 \mathrm{~mm}$ before the start of the intervention; (9) individuals who are already hospitalised; (10) individuals whose participation has been judged by a doctor as undesirable, and (11) individuals who have difficulty reading, writing or conversing in Japanese.

For participant recruitment, the study staff will first view information from the medical records of pregnant women scheduled to give birth at the National Center for Child Health and Development after they have undergone a prenatal check-up at approximately 8-10 weeks' gestation, to ensure that none of the 11 exclusion criteria apply. Participants to whom none of the exclusion criteria apply will be informed of the 
Figure 1 Summary of the study design.

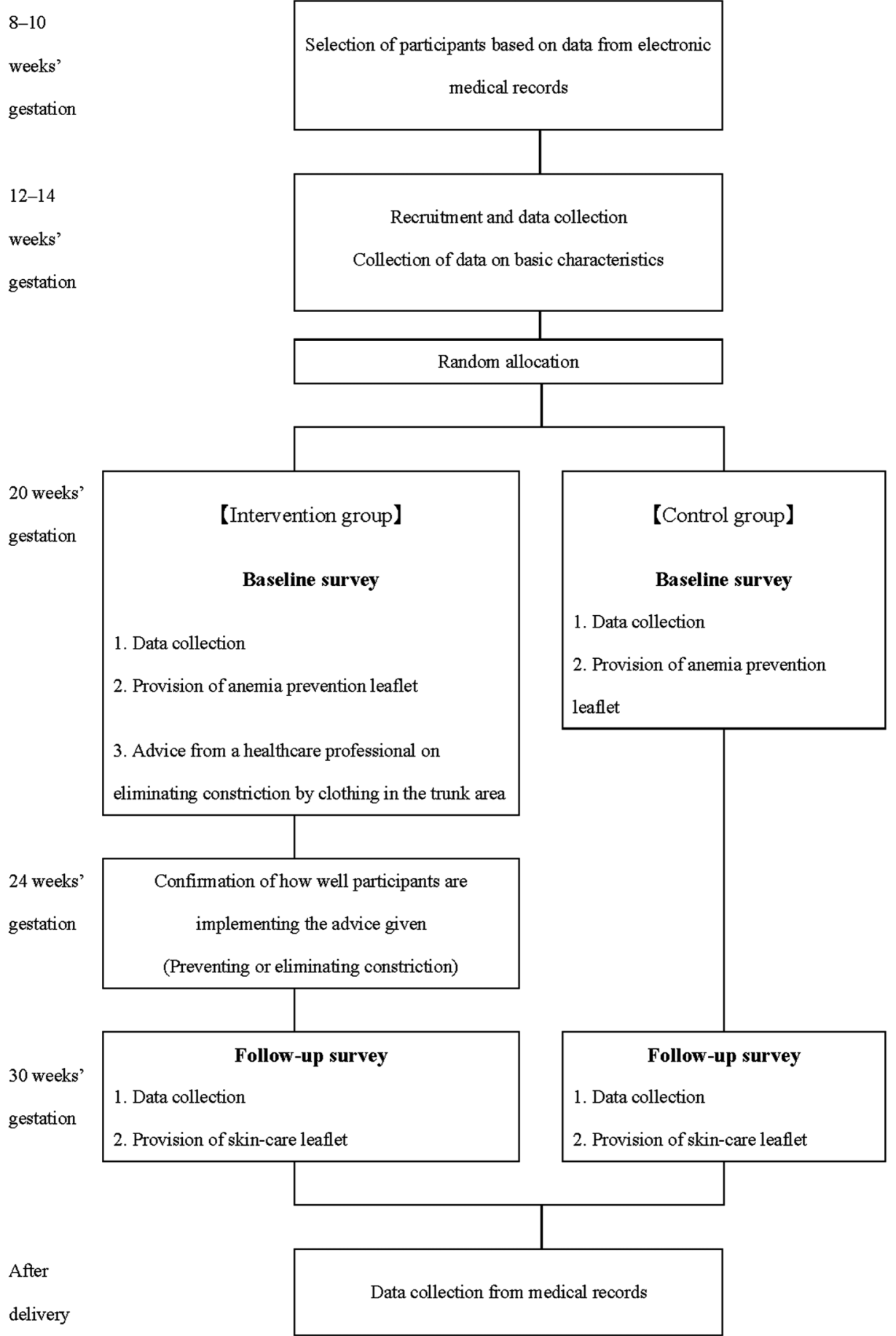

purpose of the study and its overall outline from the study staff after their prenatal check-up at approximately 12-14 weeks' gestation. At this check-up, staff will explain that non-participation in the study will not negatively affect the receipt of medical services or midwife care, and that even after they have given consent, they may still withdraw their consent. Participants who agree to participate in the study will sign a consent form, which they may either give directly to the study staff or subsequently send to the researchers by mail. Recruitment will continue until the required number of participants has been reached.

\section{Randomisation and blinding}

The RAND function in Microsoft Excel will be used to perform the permuted block method that will allocate participants to the two groups. Doctors and clinical nursing staff in the hospital who evaluate the primary outcomes of this study will be blinded to the results of this allocation. Participants cannot be blinded because the intervention programmes vary depending on the group. Participants will also be asked to avoid talking to other pregnant women about the content of the intervention programme as much as possible to prevent contamination. The study staff will be responsible for 
distributing and collecting questionnaires from participants and for performing a number of measurements. We will determine fixed procedures in advance and request that the study staff implement these in order to control performance bias.

\section{Intervention}

\section{Anaemia prevention and skin-care leaflets}

Leaflets on anaemia prevention and skin care will be distributed to participants in the intervention and control groups. The participants will receive the anaemia prevention leaflet at 20 weeks' gestation and the skin-care leaflet at 30 weeks' gestation from study staff in a private room designated for study participants. After receiving the leaflets, participants will have the opportunity to ask members of the study staff questions regarding the contents of the leaflets. The time required for implementation of the intervention programme is approximately 5 min. The main content of the anaemia prevention leaflet will consist of a basic knowledge of anaemia, the impact of anaemia on maternal and fetal health, and ways to prevent the condition. The skin-care leaflet will cover a basic knowledge of skin conditions during pregnancy, including faster growth of facial and body hair, an increase in freckles, and ways to prevent itching and stretch marks.

\section{Constriction elimination programme}

Healthcare professionals will provide this programme only to participants in the intervention group after the anaemia prevention is distributed at 20 weeks' gestation. The study staff will explain the content of the programme orally aided by illustrations and demonstrations, and will answer questions from participants. The time required for implementation of the programme is approximately $10-15 \mathrm{~min}$. The main content of the programme will consist of (1) physical changes during pregnancy; (2) potential risks that result from constricting the trunk; (3) types of clothing and how to wear them so as not to constrict the trunk; and (4) how to wear a seat belt when travelling by car. At 24 and 30 weeks' gestation, healthcare professionals will assess intervention-group participants in order to check their trunk for pressure marks on the skin and abdominal tension and to assess whether women have experienced any discomfort due to restrictive clothing. Healthcare professionals will also ask participants at 30 weeks' gestation to complete an open-ended questionnaire about their experience of this programme.

Women will be given the following information in detail. During pregnancy, hormonal effects cause the ligaments to loosen, and the shape of the pelvis and chest in particular change to support the uterus as it increases in size. Wearing clothing that constricts the trunk during pregnancy hinders these physical changes from progressing and can cause physical discomfort. Constriction above the uterus causes the uterus to be pressed against the backbone, while constriction in the chest region makes breathing more difficult and prevents the chest from expanding. It is therefore important to avoid clothing that constricts the trunk at any stage of pregnancy, so as not to inhibit natural changes in body shape.

In terms of practical clothing choices and how they should be worn, participants will be instructed to wear loose clothing overall, both as underwear and outer clothing. We will define constrictive clothing as clothing that feels tight and uncomfortable to wear, leaves pressure marks on the skin, and clothing that when removed makes the woman feel more comfortable. For example, clothing tied with ribbons around the trunk, as well as tops with built-in brassieres and tank tops, may become tight and cause constriction. Participants will be encouraged to practice caution with these garments. They will be told to avoid brassieres that feel tight and to take preventative measures, such as hooking the fastener of their bra one hook less than usual in order to give a looser fit. Even if briefs or outer clothing do not feel tight, if the elastic or other material leaves a pressure mark on the skin, they should be considered constrictive. Participants will also be told that even the design of underwear and outer clothing sold as maternity wear should be carefully regarded, and that they must be careful when purchasing clothing as some such items are structured to compress the trunk more than necessary. To keep their trunks warm, participants will be encouraged to use non-constricting belly bands, rather than adjusting their underwear or clothing.

Ligaments throughout the body become looser during pregnancy; however, loosening of the pelvis, in particular, causes a range of uncomfortable symptoms. To address these symptoms, participants will be advised to ensure that the elastic of their pants and briefs does not come up to the abdomen, but sits above the pubic bone at the hipline, thus supporting excessive loosening.

Advice on how to wear a seat belt when travelling by car during pregnancy will be provided based on the guidelines for obstetrical practice published by the Japan Society of Obstetrics and Gynecology. ${ }^{20}$ In practical terms, this means always wearing the shoulder and lap belts and ensuring that they do not cut across the bump of the pregnant uterus.

\section{Outcome measurements}

\section{Primary outcome}

The primary outcome of this study will be whether any of the following category variables has been met: (1) cervical length $<30 \mathrm{~mm}$ up to 28 weeks' gestation, (2) admission to hospital for threatened premature delivery or (3) premature delivery (gestational age $<37$ weeks). Data from all three categories will be collected from medical records by a blinded gynaecologist. A cervical length of $<30$ or $<26 \mathrm{~mm}$ is associated with a 3.8-fold or 6.2-fold relative risk of premature birth, respectively. ${ }^{19}$ The negative predictive values of a cervical length of $\geq 30 \mathrm{~mm}$ at 24 and 28 weeks' gestation are $97 \%$ and 
98\%, respectively. ${ }^{21}$ Adjusted ORs and a $95 \%$ CI of 0.95 (0.92 to 0.98$)$ and $0.94(0.92$ to 0.97$)$ were used for cervical length at 24-26 and 30-31 weeks for premature delivery. ${ }^{22}$ Considering this gynaecological evidence, the criterion of whether or not cervical length is $<30 \mathrm{~mm}$ at up to 28 weeks' gestation is considered to be valid for investigating the efficacy of advice from healthcare professionals for pregnant women.

\section{Secondary outcomes}

The secondary outcomes of this study comprise data collected for the following 10 items associated with the QoL and state of health of pregnant women: (1) tension and hardness of the abdominal wall; (2) temperature of the extremities and trunk region; (3) stress; (4) urinary incontinence; (5) QoL; (6) location of fetal activity; (7) bowel evacuation problems; (8) gynaecological complications; (9) velocity of the umbilical artery; and (10) birth weight.

Tension and hardness of the abdominal wall will be measured with a muscle hardness meter at two predetermined locations in the abdominal region, and the means of three separate measurements will be calculated in each case. Temperature of the extremities and the trunk will be measured by thermography. Stress will be measured using a salivary amylase. Urinary incontinence will be assessed using the Japanese version of the International Consultation on Incontinence Questionnaire-Short Form (ICIQ-SF). ${ }^{23}$ The QoL will be evaluated using the Japanese version of the SF-8. ${ }^{24}$ The location of fetal activity and the frequency of bowel evacuation will be ascertained from the answers to questions on subjective frequency and status in a selfadministered questionnaire. Data on these seven items will be collected by the study staff between 20 and 30 weeks' gestation. Data on the other three items (gynaecological complications, velocity of the umbilical artery and birth weight) will be transcribed by study staff from electronic medical records after delivery.

\section{Sample size calculation}

When calculating the sample size, the level of significance $(\alpha)$ will be set at 0.05 and the power $(1-\beta)$ at 0.8 . Our previous retrospective study based on data from medical records included pregnant women who were admitted to hospital for threatened premature birth, unlike the pregnant women of this study. ${ }^{20}$ Nevertheless, the study found an $8.5 \%$ difference in the incidence of premature birth at $\leq 34$ weeks' gestation, with rates of $8.7 \%$ in the intervention group and $17.2 \%$ in the nonintervention group. In practice, we anticipate the incidence of threatened premature birth at $\leq 34$ weeks' gestation to be lower than this value, given that the participants of this study will comprise a lower risk population; however, the incidence between groups will indicate the difference made by the intervention.

We used STATA12 to calculate the sample size based on this assumption, as well as the assumption that the
$8.5 \%$ difference in incidence based on medical record observations from our previous retrospective study will be obtained. If the incidence in the intervention group is assumed to be $8.1 \%$ (6\% difference in incidence), the sample size of a single group should be 462 pregnant women. For an incidence of $7.1 \%$ ( $7 \%$ difference in incidence), the sample size should be 331 women, and for an incidence of $6.1 \%$ (8\% difference in incidence) it should be 246 women. In this study, we expect to achieve an effect close to the $8.5 \%$ found in our previous study, and estimate that 246 members in each group will be required to obtain a total sample of 492 women. This will correspond to the $6.1 \%$ incidence of the primary outcome in the control group. As we envisage a dropout rate of $20 \%$ of participants between the beginning and end of the study, we calculate that 616 participants (308 in each group) will be required for this study.

\section{Handling of adverse events}

In the event of the occurrence or suspected occurrence of any serious adverse events in association with this study, in accordance with the guidelines set out by the researchers' institution, these events shall immediately be reported in writing to the Chair of the Ethics Committee and the director of the institution concerned. These members will issue advice on whether or not the study will be permitted to proceed. The level of physical invasiveness in this study is extremely low as the intervention consists of reducing clothing compression around the trunk, and data measurements will consist only of the transcription of data from self-administered questionnaires and electronic medical records, muscle hardness meter and salivary component measurements, and temperature measurement by thermography. Therefore, we believe that there is a low probability that this intervention will directly cause markedly severe adverse events among participants.

\section{Data management}

All the data collected in this study will be entered and saved on a computer that is isolated from the Internet. All data will be backed up on a password-protected device, and this device will be stringently managed. Only designated members will be able to access the study data. Paper-based documentation, such as consent forms collected during recruitment and questionnaires, will be stored until 31 March 2017 (the end of the study period), after which they will be shredded and disposed of. Electronic data from which individuals can be identified will similarly be deleted on 31 March 2017, but anonymised data sets will be stored long-term and used for generating research results.

\section{Statistical analysis}

Data analyses will be performed according to the intention-to-treat approach. Baseline data at 20 weeks of pregnancy will be compared between the intervention and control groups to assess the validity of the 
randomisation process. We will use the $\chi^{2}$ test for binary valuables, including primary outcomes regarding preterm birth, and Student's t test or the Mann-Whitney U-test for continuous valuables to evaluate the difference between the two groups, with a significance level of 0.05 . To perform multivariate analysis, we will use multiple logistic regressions for binary outcomes and multiple linear regressions for continuous outcomes. The regression analyses in this study will be adjusted for any baseline differences and other relevant covariates. The data will be analysed using STATA V.12.0 (Stata Corporation, College Station, Texas, USA) and SPSS statistics V.19.0 (IBM SPSS Statistics for Windows, IBM Corp, Armonk, New York).

Since previous studies are not sufficient to calculate the sample size of this study accurately, we will conduct an interim analysis when 100 participants from each group finish this trial. This analysis will estimate the proportion of primary and secondary outcomes in each group and calculate the sample size of this study. However, if the results of the interim analysis show no difference in any outcomes in both groups, we will consider the discontinuation of this study.

\section{Monitoring}

A Data Monitoring Board is not needed because the intervention programme of this study produces minimal risk to participants. We will conduct an interim analysis when the number of participants in both groups reaches 100 women. However, the aim of this interim analysis is not for early stopping but to redesign the sample size.

\section{ETHICS AND DISSEMINATION}

This study is of minimal risk for pregnant women because the intervention programmes regarding health advice and leaflets are not invasive. The study staff will explain the objectives and schedule of this study to all participants at recruitment. Participants who do not meet the study criteria or do not give informed written consent will not be enrolled in this study. This study has been approved by the Institutional Review Board and Ethics Committee of the National Center for Child Health and Development in Japan (Approval Number 814). Written consent to participate in this study will be obtained from all eligible participants. The findings of this study will be disseminated widely through peerreviewed publications and presented at national and international conferences.

\section{DISCUSSION}

As described above, previous research remains limited on types of clothing for pregnant women and how clothing should be worn. ${ }^{7-10} 151619$ This study is designed to verify the effect of a unique intervention programme, that is, advice from healthcare professionals to pregnant women about types of clothing and how to wear them to reduce the risk of premature birth and improve QoL during pregnancy. This programme's advantages over other premature birth prevention programmes include a low level of invasiveness and easy, cost-effective provision, without the need for special equipment. This programme could thus be adopted into clinical practice in developed countries, as well as in developing countries and rural areas with few medical resources. In addition to demonstrating the effectiveness of such advice from healthcare professionals, the implementation of this randomised comparative study may further highlight suitable types of clothing and wearing styles for pregnant women, thus potentially improving their state of health.

Previous studies on types of clothing for pregnant women and how clothing should be worn have been inadequate, and the possibility that the calculated sample size may not be appropriate cannot therefore be ruled out. However, on the basis of the results of a previous retrospective study of data from medical records as well as inferences about physiological causal relationships used in other previous studies, we consider that sufficient evidence is present to indicate that constriction of the trunk has an adverse effect on health.

\section{Author affiliations}

${ }^{1}$ Department of Health Policy, National Center for Child Health and Development, Tokyo, Japan

${ }^{2}$ Department of Nursing, National Center for Child Health and Development, Tokyo, Japan

${ }^{3}$ Division of Obstetrics, Center of Maternal-Fetal, Neonatal and Reproductive Medicine, National Center for Child Health and Development, Tokyo, Japan ${ }^{4}$ Division of Bioethics, National Center for Child Health and Development,

Tokyo, Japan

${ }^{5}$ Department of Education for Clinical Research, National Center for Child Health and Development, Tokyo, Japan

Acknowledgements The authors appreciate the help received from $\mathrm{Dr}$ Noriyoshi Watanabe, who contributed greatly to the preliminary retrospective study of medical records and the development and design of this study. They acknowledge the advice and support from medical staff and pregnant women who participated in pilot tests in this centre. The authors also appreciate the editorial support of Emma Barber of the Department of Education for Clinical Research at the National Center for Child Health and Development, Tokyo, Japan.

Contributors SK developed the intervention programmes in this study. KT, $\mathrm{SK}, \mathrm{CN}, \mathrm{AS}$ and JSC conducted the preliminary retrospective study using medical records and analysed the data. KT, SK, AS, JSC, NK, CN, HS, YN, TA, $\mathrm{El}$ and YI participated in the design of this study. KT, SK, NK, HS, YN, TA and $\mathrm{YI}$ constructed the logistics of this study. KT and SK mainly wrote the first draft of the manuscript, and all authors reviewed and revised the manuscript and gave final approval for publication of this protocol. All contributors will have the right to access the final data set of this study.

Funding This work was supported by a grant from the National Center for Child Health and Development, Tokyo, Japan (grant number: 26-8).

\section{Competing interests None declared.}

Ethics approval The study protocol has been approved by the Institutional Review Board and Ethics Committee of the National Center for Child Health and Development in Japan (approval number 814).

Provenance and peer review Not commissioned; externally peer reviewed.

Open Access This is an Open Access article distributed in accordance with the Creative Commons Attribution Non Commercial (CC BY-NC 4.0) license, which permits others to distribute, remix, adapt, build upon this work noncommercially, and license their derivative works on different terms, provided 
the original work is properly cited and the use is non-commercial. See: http:// creativecommons.org/licenses/by-nc/4.0/

\section{REFERENCES}

1. Beck S, Wojdyla D, Say $L$, et al. The worldwide incidence of preterm birth: a systematic review of maternal mortality and morbidity. Bull World Health Organ 2010;88:31-8.

2. Han S, Crowther CA, Moore V. Magnesium maintenance therapy for preventing preterm birth after threatened preterm labour. Cochrane Database Syst Rev 2013;5:CD000940.

3. Nanda K, Cook LA, Gallo MF, et al. Terbutaline pump maintenance therapy after threatened preterm labor for preventing preterm birth. Cochrane Database Syst Rev 2002;(4):CD003933.

4. Rafael TJ, Berghella V, Alfirevic Z. Cervical stitch (cerclage) for preventing preterm birth in multiple pregnancy. Cochrane Database Syst Rev 2014;9:CD009166.

5. Sosa C, Althabe F, Belizan J, et al. Bed rest in singleton pregnancies for preventing preterm birth. Cochrane Database Syst Rev 2004;3:CD003581.

6. Khianman B, Pattanittum P, Thinkhamrop J, et al. Relaxation therapy for preventing and treating preterm labour. Cochrane Database Syst Rev 2012;8:CD007426.

7. Takasu N, Furuoka S, Inatsugi N, et al. The effects of skin pressure by clothing on whole gut transit time and amount of feces. J Physiol Anthropol Appl Human Sci 2000;19:151-6.

8. Miyatsuji A, Matsumoto T, Mitarai S, et al. Effects of clothing pressure caused by different types of brassieres on autonomic nervous system activity evaluated by heart rate variability power spectral analysis. J Physiol Anthropol Appl Human Sci 2002;21:67-74

9. Sone $\mathrm{Y}$, Kato N, Kojima Y, et al. Effects of skin pressure by clothing on digestion and orocecal transit time of food. J Physiol Anthropol Appl Human Sci 2000;19:157-63.

10. Eungpinichpong W, Buttagat V, Areeudomwong $\mathrm{P}$, et al. Effects of restrictive clothing on lumbar range of motion and trunk muscle activity in young adult worker manual material handling. Appl Ergon 2013;44:1024-32.

11. Cullen G, O'Donoghue D. Constipation and pregnancy. Best Pract Res Clin Gastroenterol 2007;21:807-18.

12. Nakamura S. "Sensitivity to cold": a concept analysis. Nihon Kango Kagakkaishi [J Japan Acad Nurs Sci] 2010;30:62-71 [in Japanese].
13. Nakamura S, Horiuchi S. Relationship between advanced maternal age, hiesho (sensitivity to cold) and abnormal delivery in Japan. Open Nurs J 2013;7:142-8.

14. Nakamura S, Horiuchi S, Yanai K. [Relationhip between pregnant women's sensitivity to cold (hiesho) and premature labor assessed using propensity scores for adjusting confounding factors]. Nihon Koshu Eisei Zasshi 2012;59:381-9.

15. Nakamura S, Horiuchi S. Relationship between pregnant women's sensitivity to cold "hiesho" and abnormal delivery. J Japan Acad Midwifery 2013;27:94-9.

16. Hobel CJ, Castro L, Rosen D, et al. The effect of thigh-length support stockings on the hemodynamic response to ambulation in pregnancy. Am J Obstet Gynecol 1996;174:1734-40; discussion 40-1.

17. Hofmeyr GJ, Kulier R. Abdominal decompression in normal pregnancy. Cochrane Database Syst Rev 2012;6:CD001062.

18. Hofmeyr GJ. Abdominal decompression for suspected fetal compromise/pre-eclampsia. Cochrane Database Syst Rev 2012;6: CD000004.

19. Kato S, Takehara K, Nitta C, et al. Advice from healthcare providers to pregnant women admitted for threatened miscarriage or premature birth to avoid wearing constrictive clothing during pregnancy: a retrospective study. Nihon Josan Gakkaishi [J Japan Acad Midwifery] 2015;28:498 [in Japanese].

20. Minakami H, Hiramatsu $\mathrm{Y}$, Koresawa $\mathrm{M}$, et al. Guidelines for obstetrical practice in Japan: Japan Society of Obstetrics and Gynecology (JSOG) and Japan Association of Obstetricians and Gynecologists (JAOG) 2011 edition. J Obstet Gynaecol Res 2011;37:1174-97.

21. lams JD, Goldenberg RL, Meis PJ, et al. The length of the cervix and the risk of spontaneous premature delivery. National Institute of Child Health and Human Development Maternal Fetal Medicine Unit Network. N Engl J Med 1996;334:567-72.

22. Hiersch L, Yogev $Y$, Domniz N, et al. The role of cervical length in women with threatened preterm labor: is it a valid predictor at any gestational age? Am J Obstet Gynecol 2014;211:532.e1-9.

23. Avery K, Donovan J, Peters TJ, et al. ICIQ: a brief and robust measure for evaluating the symptoms and impact of urinary incontinence. Neurourol Urodyn 2004;23:322-30.

24. Ware JE, Kosinski M, Dewey J, et al. How to score and interpret single-item health status measures: a manual for users of the SF-8 health survey. Boston: QualyMetric, 2001. 